\title{
Phase I trials in patients with relapsed, advanced upper gastrointestinal carcinomas: experience in a specialist unit
}

\author{
Khurum Khan · Joo Ern Ang • Naureen Starling • Francesco Sclafani • \\ Krunal Shah • Ian Judson • L. Rhoda Molife - Udai Banerji · Johann S. de Bono • \\ David Cunningham • Stan B. Kaye
}

Received: 14 May 2013/Accepted: 16 December 2013/Published online: 21 January 2014

(C) The International Gastric Cancer Association and The Japanese Gastric Cancer Association 2014

\begin{abstract}
Background Conventional therapeutic options for patients with advanced upper gastrointestinal cancers (UGIC) are limited. Following first-line treatments, some patients are offered experimental therapies, including participation in Phase I trials. This study aims to describe the experience of UGIC patients treated in a dedicated Phase I unit.

Methods Patient, tumour and treatment characteristics, and clinical outcomes of UGIC patients treated consecutively at the Drug Development Unit, Royal Marsden Hospital, between 2005 and 2009, were recorded.

Results Ninety-six patients who previously received a median of 2 (range 1-4) lines of chemotherapies were treated in 30 Phase I trials. Of 81 evaluable patients, 9
\end{abstract}

K. Khan and J.E. Ang contributed equally to this work and are joint first authors.

K. Khan · J. E. Ang $\cdot$ N. Starling $\cdot$ K. Shah $\cdot$ I. Judson

L. R. Molife · U. Banerji · J. S. de Bono · S. B. Kaye (ه)

Drug Development Unit, Sycamore House, The Royal Marsden

NHS Foundation Trust, Sutton SM2 5PT, UK

e-mail: Stan.Kaye@rmh.nhs.uk

K. Khan · N. Starling $\cdot$ F. Sclafani $\cdot$ D. Cunningham Gastrointestinal Unit, The Royal Marsden NHS Foundation

Trust, Sutton SM2 5PT, UK

e-mail: khurum.khan@icr.ac.uk

J. E. Ang · U. Banerji · J. S. de Bono - S. B. Kaye Division of Cancer Therapeutics, The Institute of Cancer Research, Sutton SM2 5NG, UK

J. E. Ang · L. R. Molife · U. Banerji · J. S. de Bono ·

S. B. Kaye

Division of Clinical Studies, The Institute of Cancer Research,

Sutton SM2 5NG, UK achieved RECIST-objective response (11\%) with a 6-month clinical benefit rate of $14 \%$. Median progression free and overall survival were 7.7 weeks [95 \%CI 7.7 (6.4-9.0)] and 19.1 weeks (95\%CI 17.5-20.8), respectively. Grade 3 or 4 toxicities were observed in 37 patients (39\%) and led to trial discontinuation in 9 (9\%); no toxicity-related death was recorded. In the multivariate analysis, serum albumin $(<35 \mathrm{~g} / \mathrm{dl}$, HR2.0, $p=0.002)$ and lactate dehydrogenase $(>192 \mu \mathrm{mol} / \mathrm{l}, \mathrm{HR} 1.7, p=0.016)$ were prognostic of overall survival.

Conclusion Phase I clinical trials can be considered a reasonable option in selected patients with relapsed UGIC. The use of objective prognosticators may improve selection and risk/benefit profile of patients.

Keywords Experimental therapy - Oesophago-gastric cancer - Pancreatic cancer - Prognosticator of overall survival (OS) · Phase I trials · Upper GI cancers (UGIC)

\section{Introduction}

Oesophago-gastric cancer (OGC) and pancreatic cancer (PC) are the second and fourth most common cause of cancer-related death in the world, respectively [1]. Both diseases often present in advanced stages and, due to their relative chemo-resistance and the limited availability of effective treatment strategies, are associated with poor survival [2, 3]. Only a minority of patients qualify for potentially curative surgery and, even in these patients, the outcome is poor with expected 5-year survival rates of approximately $37 \%$ for OGC in Western countries and $<20 \%$ for PC $[4,5]$.

Palliative chemotherapy has demonstrated improvement in overall survival and clinical benefit compared to best supportive care (BSC) and represents the standard 
treatment for patients with relapsed or metastatic disease with OGC and PC [6, 7]. Doublet or triplet combination chemotherapy regimens are now widely used in patients with a good performance status (PS) [8-10]. Moreover, targeted agents, including trastuzumab in HER-2 positive OGC patients and erlotinib in unselected PC patients, have been included in the therapeutic armamentarium for these diseases [11, 12].

In contrast with the first-line setting, limited data on the role of second-line therapies for OGC and PC have been historically available and, given the modest benefit of such therapies associated with the poor performance of chemorefractory patients, chemotherapy has not been routinely delivered especially in Western countries [13-15]. More recently, two Phase III trials showed a survival advantage for salvage chemotherapy (taxanes or irinotecan) compared with BSC [16] in chemo-refractory OGC, supporting the potential utility of salvage treatments in this setting. Less data are available for chemo-refractory PC. Second line therapies have been investigated in Phase II trials with limited success [17-21], and there is currently no standard regimen for these patients.

The limited availability of standard effective treatments coupled with the advances in the understanding of tumour biology and the development of molecularly targeted therapies have progressively increased the number of chemo-refractory OGC and PC patients who are referred for consideration of Phase I trials.

Investigating the safety of new agents and finding a dose to further test in the subsequent stages of the drug development process are the main purposes of Phase I trials. However, there is a lack of published data regarding the impact of experimental drug therapies on the safety and outcome of chemo-refractory OGC and PC patients. We therefore conducted a retrospective analysis of all patients with UGIC treated in the specialist Phase I unit at our institution. The primary aim of this study was to describe the rates of grade 3 or 4 toxicities and treatment-related trial discontinuation in these patients, as well as defining standard efficacy parameters. We additionally explored the prognostic role of baseline variables for this group of patients.

\section{Patient and methods}

This retrospective study comprised all patients with UGIC consecutively treated in Phase I clinical trials at the Drug Development Unit, Royal Marsden National Health Service (NHS) Foundation Trust, Sutton, United Kingdom, from January 2005 to December 2009. Only patients who received at least one dose of the experimental agent were included. Clinical parameters prospectively collected for this study included site of origin of the primary tumour, histological subtype, age, gender, Eastern Cooperative Oncology Group (ECOG) PS, full blood count, biochemical profiles [including alkaline phosphatase (ALP), bilirubin, lactate dehydrogenase (LDH) and albumin], number of metastatic sites, and number of lines of previous systemic therapies. All study patients had previously provided written informed consent for participation in the relevant Phase I trials as approved by the local Research Ethics Committees.

Toxicity assessment and response evaluation

Toxicity data were collected as originally recorded in the electronic medical records (EMR), and when required, from the case report forms (CRFs). In all trials included in the present analysis, toxicity was graded according to the Common Terminology Criteria for Adverse Events (CTCAE) version 2.0 or 3.0.

Baseline tumour measurements were performed within 4 weeks prior to treatment start. Tumour measurements were repeated every 6-8 weeks during the first 6 months on trial using Response Evaluation Criteria In Solid Tumours (RECIST) version 1.0. Tumour responses were confirmed prospectively by a radiologist. Survival data were obtained from the hospital EMR, and when necessary, by contacting the general practitioner or referring institution.

\section{Statistical methods}

OS was defined as the interval between the day of first administration of the experimental therapy and the date of death from any cause. Patients who were lost to follow-up were censored at the date of last contact. Progression-free survival (PFS) was defined by the time elapsed between Cycle 1 day 1 on study until radiological progression or death of any cause while on treatment (which ever occurred first); if no evidence of progression was documented at the last follow-up, patients were censored at the time of last radiological evaluation. The clinical benefit rate (CBR) was calculated as the sum of objective response rate $(\mathrm{ORR})$ and proportion of patients with stable disease (SD) at 4 or 6 months.

Categorisation of numeric variables was undertaken based on considerations of the standard reference values (normal range versus low/elevated) or according to the median values. Estimates of median PFS and OS [and $95 \%$ confidence interval $(\mathrm{CI})]$ were determined using the Kaplan-Meier method, and survival curves were compared using the log-rank test for categorical variables. Cox regression analysis was undertaken to identify baseline characteristics that provided prognostic information. 
Table 1 Patient and tumour characteristics

\begin{tabular}{|c|c|c|c|}
\hline Characteristic & OG & Pancreatic & All \\
\hline Age, years (median, range) & $58(24-77)$ & $63(39-75)$ & $59(24-77)$ \\
\hline Albumin, g/l (median, range) & $33(20-39)$ & $35(24-42)$ & $34(20-42)$ \\
\hline Alkaline phosphatase, $\mu \mathrm{mol} / \mathrm{l}$ (median, range) & $95(43-2483)$ & $146(68-754)$ & $100(43-2483)$ \\
\hline Bilirubin, $\mu \mathrm{mol} / 1$ (median, range) & $12(4-30)$ & $10(6-29)$ & $11(4-30)$ \\
\hline Lactate dehydrogenase (median, range) & $158(71-1815)$ & $140(112-392)$ & $151(71-1815)$ \\
\hline WCC, $10^{3} / \mathrm{mm}^{3}$ (median, range) & $8(2.9-29)$ & $6.9(3.5-10.2)$ & $7.6(2.9-29)$ \\
\hline Lymphocyte, $10^{3} / \mathrm{mm}^{3}$ (median, range) & $1.1(3-4.2)$ & $1.0(0.3-4.2)$ & $1.1(0.3-4.2)$ \\
\hline Neutrophil, $10^{3} / \mathrm{mm}^{3}$ (median, range) & $6.1(1.7-28.1)$ & $4.8(1.8-7.0)$ & $5.7(1.7-28.1)$ \\
\hline Haemoglobin, g/dl (median, range) & $11.8(9.2-15.1)$ & $12(10.3-15.3)$ & $12(9.2-15.3)$ \\
\hline Platelets, $10^{3} / \mathrm{mm}^{3}$ (median, range) & $287(110-712)$ & $231(146-443)$ & $285(110-712)$ \\
\hline \multicolumn{4}{|l|}{ Gender $(n, \%)$} \\
\hline Male & $58(82)$ & $17(68)$ & $75(78)$ \\
\hline Female & $13(18)$ & $8(8)$ & $21(22)$ \\
\hline \multicolumn{4}{|l|}{ ECOG PS $(n, \%)$} \\
\hline 0 & $16(23)$ & $10(40)$ & $26(27)$ \\
\hline 1 & $52(73)$ & $13(52)$ & $65(68)$ \\
\hline 2 & $2(3)$ & $2(8)$ & $4(4)$ \\
\hline Not known & $1(1)$ & $0(0)$ & $1(1)$ \\
\hline \multicolumn{4}{|l|}{ No. of metastatic sites $(n, \%)$} \\
\hline 0 & $2(3)$ & $1(4)$ & $3(3)$ \\
\hline 1 & $16(23)$ & $8(32)$ & $24(25)$ \\
\hline 2 & $36(51)$ & $9(36)$ & $45(47)$ \\
\hline 3 & $13(18)$ & $4(16)$ & $17(18)$ \\
\hline 4 & $4(6)$ & $3(12)$ & $7(7)$ \\
\hline \multicolumn{4}{|l|}{ Histology $(n, \%)$} \\
\hline Adenocarcinoma & $54(76)$ & $20(80)$ & $74(77)$ \\
\hline Squamous & $10(14)$ & $0(0)$ & $10(10)$ \\
\hline Others & $1(1)$ & $2(8)$ & $3(3)$ \\
\hline Not known & $6(8)$ & $3(12)$ & $9(9)$ \\
\hline \multicolumn{4}{|l|}{ No. of previous lines of systemic therapy $(n, \%)$} \\
\hline 1 & $32(45)$ & $8(32)$ & $40(42)$ \\
\hline 2 & $29(41)$ & $12(48)$ & $41(43)$ \\
\hline 3 & $9(13)$ & $4(16)$ & $13(14)$ \\
\hline 4 & $1(1)$ & $0(0)$ & $1(1)$ \\
\hline Not known & $0(0)$ & $1(4)$ & $1(1)$ \\
\hline \multicolumn{4}{|l|}{ Chemotherapy containing regimen $(n, \%)$} \\
\hline Yes & $31(44)$ & $4(16)$ & $35(36)$ \\
\hline No & $40(56)$ & $21(84)$ & $61(64)$ \\
\hline \multicolumn{4}{|l|}{ Chemotherapy regimen utilised $(n, \%)$} \\
\hline Carboplatin/paclitaxel & $9(29)$ & $2(50)$ & $11(31)$ \\
\hline Docetaxel & $16(52)$ & 0 & $16(46)$ \\
\hline Paclitaxel & $2(6)$ & 0 & $2(6)$ \\
\hline Gemcitabine & $3(10)$ & 0 & $3(9)$ \\
\hline Doxorubicin & $1(3)$ & 0 & $1(3)$ \\
\hline Cyclophosphamide & $0(0)$ & $2(50)$ & $2(6)$ \\
\hline \multicolumn{4}{|l|}{ Best RECIST-objective response $(n, \%)$} \\
\hline Progressive disease & $28(39)$ & $13(52)$ & $41(43)$ \\
\hline Stable disease & $24(34)$ & $7(28)$ & $31(32)$ \\
\hline
\end{tabular}


Table 1 continued

\begin{tabular}{llll}
\hline Characteristic & OG & Pancreatic & All \\
\hline Partial response & $9(13)$ & $0(0)$ & $9(9)$ \\
Not evaluable & $10(14)$ & $5(20)$ & $15(16)$ \\
CBR at 4 months $(n, \%)$ & $15(21)$ & $2(8)$ & $17(18)$ \\
Yes & $46(65)$ & $18(72)$ & $64(67)$ \\
No & $10(14)$ & $5(20)$ & $15(16)$ \\
Not evaluable & & $1(4)$ & $11(11)$ \\
CBR at 6 months $(n, \%)$ & $10(14)$ & $19(76)$ & $70(73)$ \\
Yes & $51(72)$ & $5(20)$ & $15(16)$ \\
No & $10(14)$ & \\
Not evaluable & & \\
\hline
\end{tabular}

Variables with a $p$ value $\leq 0.10$ were entered in a multivariate model, where a forward stepwise approach was taken. $p$ Values $<0.05$ were considered significant.

A prognostic score was developed based on the two equally-weighted variables that were significant in the multivariate analysis, namely: LDH normal (0) versus LDH > ULN (+1); albumin more than $35 \mathrm{~g} / 1$ (0) versus albumin $<35 \mathrm{~g} / 1(+1)$. The prognostic score for each individual was derived from the sum of these two components. In addition, the performance of the new prognostic score was evaluated by calculating the area under the receiver operating characteristic curve. All $p$ values presented in this study are 2-sided. All analyses were performed using SPSS 16.0 (SPSS Inc., Chicago, Il, USA).

\section{Results}

Patient and tumour characteristics

One thousand and four patients were recruited into Phase I trials between 2005 and 2009 in 30 Phase I clinical trials in our institution; of those $96(9.5 \%)$ had UGIC. The median age at Phase I trial entry was 59 years (range 24-77) with a male/female ratio of 3.5. Of the total cohort, 71 patients $(74 \%)$ had OGC; primary carcinoma of the oesophagus (41), OGJ (7) or stomach (14), while 25 patients $(26 \%)$ had primary carcinoma of the pancreas. The median number of prior lines of chemotherapy was 2 (range 1-4). Sixty-one patients (64\%) were treated with single agent or combined experimental therapies. Fourteen percent of patients received therapy in more than one Phase I trial.

Thirty-five (36\%) patients received a chemotherapycontaining regimen; these included carboplatin-paclitaxel in $11(31 \%)$, paclitaxel in $2(6 \%)$, docetaxel in $16(46 \%)$, gemcitabine in $3(9 \%)$, cyclophosphamide in $2(6 \%)$ and
Table 2 All observed grade 3 or 4 possible or likely treated-related AEs in all study patients $(n=96)$ during dose limiting toxicitydefining period

\begin{tabular}{|c|c|c|c|c|c|}
\hline & \multicolumn{2}{|c|}{ Biologics only } & \multicolumn{2}{|c|}{$\begin{array}{l}\text { Chemotherapy } \\
\text { containing } \\
\text { studies }\end{array}$} & \multirow{2}{*}{$\begin{array}{l}\text { Any grade } \\
3 \text { or } 4 \mathrm{AE} \text { in } \\
\text { all study } \\
\text { patients }\end{array}$} \\
\hline & $\begin{array}{l}\text { Grade } \\
3 \text { AEs }\end{array}$ & $\begin{array}{l}\text { Grade } \\
4 \text { AEs }\end{array}$ & $\begin{array}{l}\text { Grade } \\
3 \text { AEs }\end{array}$ & $\begin{array}{l}\text { Grade } \\
4 \text { AEs }\end{array}$ & \\
\hline Fatigue & - & - & 3 & - & 3 \\
\hline Rash & 2 & - & - & - & 2 \\
\hline Musocitis & 1 & - & - & - & 1 \\
\hline Nausea & 2 & - & - & - & 2 \\
\hline Diarrhoea & 2 & - & - & 1 & 3 \\
\hline $\begin{array}{l}\text { Rise in hepatic } \\
\text { transaminase }\end{array}$ & 2 & - & 2 & - & 4 \\
\hline Neutropenia & 1 & 2 & 2 & 4 & 9 \\
\hline Anaemia & - & - & 1 & - & 1 \\
\hline Hyponatremia & 1 & - & - & - & 1 \\
\hline
\end{tabular}

doxorubicin in 1 (3\%). At Phase I trial entry, 65 patients (68 \%) had an ECOG PS of 1 and 26 (27\%) an ECOG PS of 0 . A summary of these results is provided in Table 1.

Safety and tolerability

Ninety-six patients received at least one dose of experimental therapy and were evaluable for toxicity assessments. Most patients developed grade 1 or 2 toxicity (71\%). Thirty-seven patients (39\%) sustained grade 3 or 4 toxicity. All grade 3 and 4 adverse events (AE) that occurred in cycle 1 deemed possibility or likely related to treatment are summarised in Table 2. No obvious difference in the serious treatment-related AE profile of the nonchemotherapy and chemotherapy-containing regimens were observed. No toxicity-related death was observed. Nine patients $(9 \%)$ discontinued trial participation due to treatment-related toxicities. 
Table 3 Efficacy estimates in study patients

\begin{tabular}{|c|c|c|c|}
\hline & Biologics only & Chemotherapy combination & All patients \\
\hline \multicolumn{4}{|l|}{ Best RECIST-responses (no. cases/evaluable, \%) } \\
\hline Progressive disease & $28 / 51(55)$ & $13 / 30(43)$ & $41 / 81(51)$ \\
\hline Stable disease & 20/51 (39) & $11 / 30(37)$ & $31 / 81(38)$ \\
\hline Partial response & $3 / 51(6)$ & $6 / 30(20)$ & 9/81 (11) \\
\hline CBR at 4 months (no. cases/evaluable, \%) & 9/61 (15) & $7 / 35(20)$ & $16 / 81(20)$ \\
\hline CBR at 6 months (no. cases/evaluable, \%) & $5 / 61(8)$ & $7 / 35(20)$ & $12 / 81(15)$ \\
\hline \multicolumn{4}{|l|}{ PFS (weeks) } \\
\hline Median $(95 \% \mathrm{CI})$ & $7.3(5.7-8.9)$ & $7.9(4.5-11.3)$ & $7.7(6.4-9.0)$ \\
\hline \multicolumn{4}{|l|}{ OS (weeks) } \\
\hline Median $(95 \% \mathrm{CI})$ & $18.4(14.1-22.8)$ & $19.6(18.4-20.8)$ & $19.1(17.5-20.8)$ \\
\hline Alive at 90 days (no. cases/evaluable, \%) & $41 / 61(67)$ & $25 / 35(71)$ & 66/96 (69) \\
\hline
\end{tabular}

\section{Efficacy}

Eighty-one patients (84\%) were evaluable for response assessment by RECIST criteria. Of these, 9 (11\%) achieved a partial response (PR) and 31 (38\%) achieved stable disease (SD). Eight out of 9 responders were in the group of OGC (6 oesophagus and 2 stomach) and one response was observed in a patient with carcinoma of unknown primary but of likely upper GI origin. There were no responses in the group of PC patients.

A median of 1.5 lines of chemotherapy were administered prior to Phase I trial participation to the patients who achieved a PR. Five patients received chemotherapy-containing regimens (docetaxel in 4 and carboplatin/paclitaxel in 1) and one received an oncolytic virus-coupled to radiotherapy; the other 3 patients received a single agent experimental therapy with a multi-tyrosine inhibitor (TKI) to vascular endothelial growth factor receptor (VEGFR) and fibroblast growth factor receptor (FGRF), a polo-like kinase (PLK) inhibitor and a monoclonal antibody targeting insulin like growth factor 1 (IGF1), respectively.

In our series, 2 patients achieved SD that lasted beyond 6 months; one patient received a chemotherapy regimen containing carboplatin-paclitaxel while the other received an AKT inhibitor as monotherapy. The efficacy estimates of patients that received biologics alone or in combination with chemotherapy are summarised in Table 3 .

The overall clinical benefit rates at 4 and 6 months were 21 and $14 \%$, respectively. Of note, $70 \%$ of patients were alive at 90 days post-Phase I trial entry. After a median follow-up of 147 weeks, the overall median PFS and OS for the overall population were 7.7 (95 \%CI 6.4-9.0) and 19.1 (17.5-20.8), respectively. When, the survival outcomes were analysed in the two cancer groups, PFS and OS in the OGC group were not found to be statistically significant.
Table 4 Details of patients who achieved RECIST responses and clinical benefit at 4 or 6 months

\begin{tabular}{|c|c|c|c|}
\hline & $\begin{array}{l}\text { RECIST } \\
\text { responders }\end{array}$ & $\begin{array}{l}\text { CBR at } \\
4 \text { months }\end{array}$ & $\begin{array}{l}\text { CBR at } \\
6 \text { months }\end{array}$ \\
\hline $\begin{array}{c}\text { Total number } \\
\text { of patients }\end{array}$ & 9 & 16 & 12 \\
\hline \multicolumn{4}{|l|}{ Primary site (no.) } \\
\hline Oesophagus & 6 & 11 & 8 \\
\hline Stomach & 2 & 2 & 2 \\
\hline Pancreas & 0 & 2 & 1 \\
\hline Unknown & 1 & 1 & 1 \\
\hline \multicolumn{4}{|c|}{ Previous lines chemotherapy (no.) } \\
\hline 1 & 3 & 4 & 4 \\
\hline 2 & 6 & 12 & 8 \\
\hline \multicolumn{4}{|c|}{ Chemotherapy containing regimen } \\
\hline Yes & 6 & 7 & 7 \\
\hline No & 3 & 9 & 5 \\
\hline \multicolumn{4}{|c|}{ Chemotherapy regimen used } \\
\hline $\begin{array}{c}\text { Carboplatin/ } \\
\text { paclitaxel }\end{array}$ & 1 & 2 & 2 \\
\hline Docetaxel & 5 & 5 & 5 \\
\hline \multicolumn{4}{|c|}{ Best RECIST response } \\
\hline Stable disease & 0 & 7 & 3 \\
\hline $\begin{array}{l}\text { Partial } \\
\text { response }\end{array}$ & 9 & 9 & 9 \\
\hline \multicolumn{4}{|l|}{ PFS (weeks) } \\
\hline $\begin{array}{l}\text { Median } \\
\quad(95 \% \mathrm{CI})\end{array}$ & $26.4(21.0-31.9)$ & $26.4(23.4-29.5)$ & $26.4(23.3-29.6)$ \\
\hline \multicolumn{4}{|l|}{ OS (weeks) } \\
\hline $\begin{array}{l}\text { Median } \\
\quad(95 \% \mathrm{CI})\end{array}$ & $51.0(16.8-85.2)$ & $54.4(44.6-64.2)$ & $50.6(36.3-64.9)$ \\
\hline
\end{tabular}

The median PFS and OS in the group of 9 patients who achieved a PR were 26.4 weeks (95\%CI 21.0-31.9) and 51.0 weeks (95\%CI 16.8-85.2), respectively. These results are summarised in Table 4. 
Table 5 Prognosticators of OS

\begin{tabular}{|c|c|c|c|c|c|}
\hline \multirow[t]{2}{*}{ Variable $(n=96)$} & \multicolumn{2}{|l|}{ Univariate analysis } & \multirow[t]{2}{*}{ Variable } & \multicolumn{2}{|l|}{ Multivariate analysis } \\
\hline & HR (95\%CI) & $p$ Value & & HR $(95 \% \mathrm{CI})$ & $p$ Value \\
\hline Age & $1.026(1.001-1.051)$ & 0.044 & Age $>65$ years & - & NS \\
\hline Pancreatic primary & $1.439(0.887-2.332)$ & 0.140 & & & \\
\hline Albumin & $0.915(0.869-0.964)$ & 0.001 & Albumin $<35 \mathrm{~g} / \mathrm{dl}$ & $2.034(1.308-3.162)$ & 0.002 \\
\hline Bilirubin & $1.028(0.996-1.062)$ & 0.091 & & & \\
\hline ALP & $1.002(1.001-1.002)$ & $<0.001$ & ALP $>110 \mu \mathrm{mol} / 1$ & - & NS \\
\hline LDH & $1.002(1.001-1.003)$ & $<0.001$ & $\mathrm{LDH}>\mathrm{ULN}$ & $1.690(1.101-2.593)$ & 0.016 \\
\hline White cell count & $1.054(0.993-1.119)$ & 0.081 & & & \\
\hline Neutrophil count & $1.063(1.005-1.125)$ & 0.033 & Neutrophil $>7,000 / \mathrm{mm}^{3}$ & - & NS \\
\hline Lymphocyte count & $0.790(0.568-1.098)$ & 0.160 & & & \\
\hline Haemoglobin & $0.940(0.804-1.101)$ & 0.444 & & & \\
\hline Platelet count & $1.000(0.999-1.002)$ & 0.722 & & & \\
\hline ECOG PS & & 0.002 & ECOG PS & - & NS \\
\hline 1 versus 0 & $2.247(1.365-3.699)$ & 0.001 & & & \\
\hline 2 versus 0 & $4.098(1.372-12.240)$ & 0.012 & & & \\
\hline No. of metastatic sites & $0.990(0.775-1.265)$ & 0.936 & & & \\
\hline Lung metastasis & $1.124(0.714-1.769)$ & 0.615 & & & \\
\hline Liver metastasis & $1.463(0.954-2.244)$ & 0.081 & Liver metastasis & - & NS \\
\hline Bone metastasis & $0.796(0.441-1.440)$ & 0.451 & & & \\
\hline Chemotherapy containing study & $1.028(0.668-1.583)$ & 0.899 & & & \\
\hline
\end{tabular}

$N S$ not significant, $U L N$ upper limit of normality

Table 6 Impact of prognostic score on OS

\begin{tabular}{llr}
\hline Prognostic score & \multicolumn{2}{l}{ OS (weeks) } \\
\cline { 2 - 3 } & Median & $95 \% \mathrm{CI}$ \\
\hline $0(n=25)$ & 28.0 & $14.5-41.5$ \\
$1(n=42)$ & 20.0 & $15.6-24.4$ \\
$2(n=29)$ & 13.0 & $9.2-16.8$ \\
Overall log-rank $p$ value & 0.001 & \\
\hline
\end{tabular}

\section{Prognosticators of OS}

Age, ECOG PS, presence of hepatic metastases, absolute neutrophil count, ALP, LDH and serum albumin were significantly associated with OS. However, in the multivariate analysis, only serum albumin $[<35 \mathrm{~g} / \mathrm{dl}, \mathrm{HR} 2.0$ (95 \%CI 1.3-3.2), $p=0.002]$ and LDH [ $>192 \mathrm{U} / 1 \mathrm{ULN}$, HR 1.7 (95 \%CI 1.1-2.6), $p=0.016]$ retained their statistical significance as baseline predictive factor of worse OS. These results are summarised in Tables 5 and 6 .

Using a prognostic model that comprised LDH and serum albumin, 3 distinct OS strata were identified: scores 0,1 and 2 with respective median OS of 28.0 (95\%CI 14.5-41.5), 20.0 (95\%CI 15.6-24.4) and 13.0 (95\%CI $9.2-16.8)$, respectively $(p=0.001)$. The Kaplan-Meier curves of OS according to scores 0-2 are shown in Fig. 1.
Using this model with mortality at 90-day as an endpoint, the area under the receiver operating characteristics curve was 0.710 (95\%CI 0.601-0.8).

\section{Discussion}

Over the last few years, the identification of new, tumourassociated genomic alterations and a better understanding of key oncogenic signalling pathways have led to a significant increase in the number of new drugs targeting these pathways in various malignancies.

Phase I trials are an essential step of a complex, multiphase drug development process where new drugs or their combinations showing promising activity in preclinical experiments are, for the first time, tested in humans with the aim of defining their safety and possibly informing on the scope of further development. However, Phase I trials also represent an attractive option for patients as well as an important resource for healthcare providers where no standard effective anti-cancer therapies are available. In this context, analysing the overall performance of Phase I trials in specific cancer subgroups, in terms of both safety and efficacy, is of interest and may also be helpful in providing useful data to inform the future directions of drug development. This is especially true for diseases like 
OGC and PC where limited therapeutic options are available on progression to first line chemotherapy, and no major, targetable oncogenic drivers (e.g., BRAF in melanoma) have been yet identified.

Very few data are currently available regarding the experience of treating patients with UGIC in the setting of Phase I clinical trials. The primary aim of our study was to analyse the safety and outcome of patients with UGIC referred to a dedicated Phase I drug development unit in a regional centre over a period of 5 years. In accordance with differences in prevalence between OGC and PC, our study population was mainly composed of OGC patients with PC patients accounting for only $26 \%$ of the cases. However, we believe that this finding may also reflect differences in natural history and standard treatment options between these malignancies, as well as potentially different patient's and physician's perceptions of the balance between treatment associated risk and gain in survival/ quality of life when discussing referral for consideration of further treatment within the setting of Phase I trials.

We showed that safety was overall acceptable in both OGC and PC patients. Indeed, in our series, the treatments were generally well tolerated with $61 \%$ of patients experiencing only grade 1 or 2 toxicities. Although $39 \%$ of patients developed grade 3 or 4 toxicities, only in $9 \%$ of cases trial discontinuation was necessary. Moreover, it is worth noting that no treatment-related deaths were observed throughout the study period. The commonest grade 3 or 4 toxicities observed in our cohort included neutropenia, fatigue, rash and diarrhoea. Interestingly, contrary to the common concern, we did not notice significant difference in the toxicity profile of patients being treated on biologics alone or in combination with chemotherapy.

When we analysed the overall outcome of the experimental agents administered, we found some drug activity with an ORR of $11 \%$ and an overall CBR at 6 months of $14 \%$. In our study, we described CBR as those patients achieving an objective response by RECIST, or stable disease for at least 4 months. However, we acknowledge that the assessment of clinical benefit in non-randomised Phase I clinical trials is difficult, since lack of disease progression, which may relate to underlying disease biology and tumour kinetics, may inappropriately be attributed to the impact of therapy. Ongoing work seeks to refine this definition through a detailed analysis of pre-treatment growth indices [22].

Forty-four percent and $16 \%$ of the OGC and PC patients, respectively, were enrolled into Phase I trials which incorporated chemotherapy. Interestingly, in our analysis, drug activity was limited to the group of patients with OGC and mainly observed when the experimental drug was administered in combination with chemotherapy. Although, this finding would support a role for chemotherapy in the refractory setting, it is difficult to ascertain the relative contribution of each treatment component. However, it is worth noting that 4 of 6 patients who were treated with chemotherapy-containing regimens also received an insulin-like growth factor 1 receptor (IGF-1R) inhibitor (in combination with docetaxel). Moreover, 1 of 3
Fig. 1 The Kaplan-Meier curves of OS according to perofrmance status scores $0-2$. Using this model with mortality at 90-day as an endpoint, the area under the receiver operating characteristics curve was 0.710 (95\%CI 0.601-0.8)

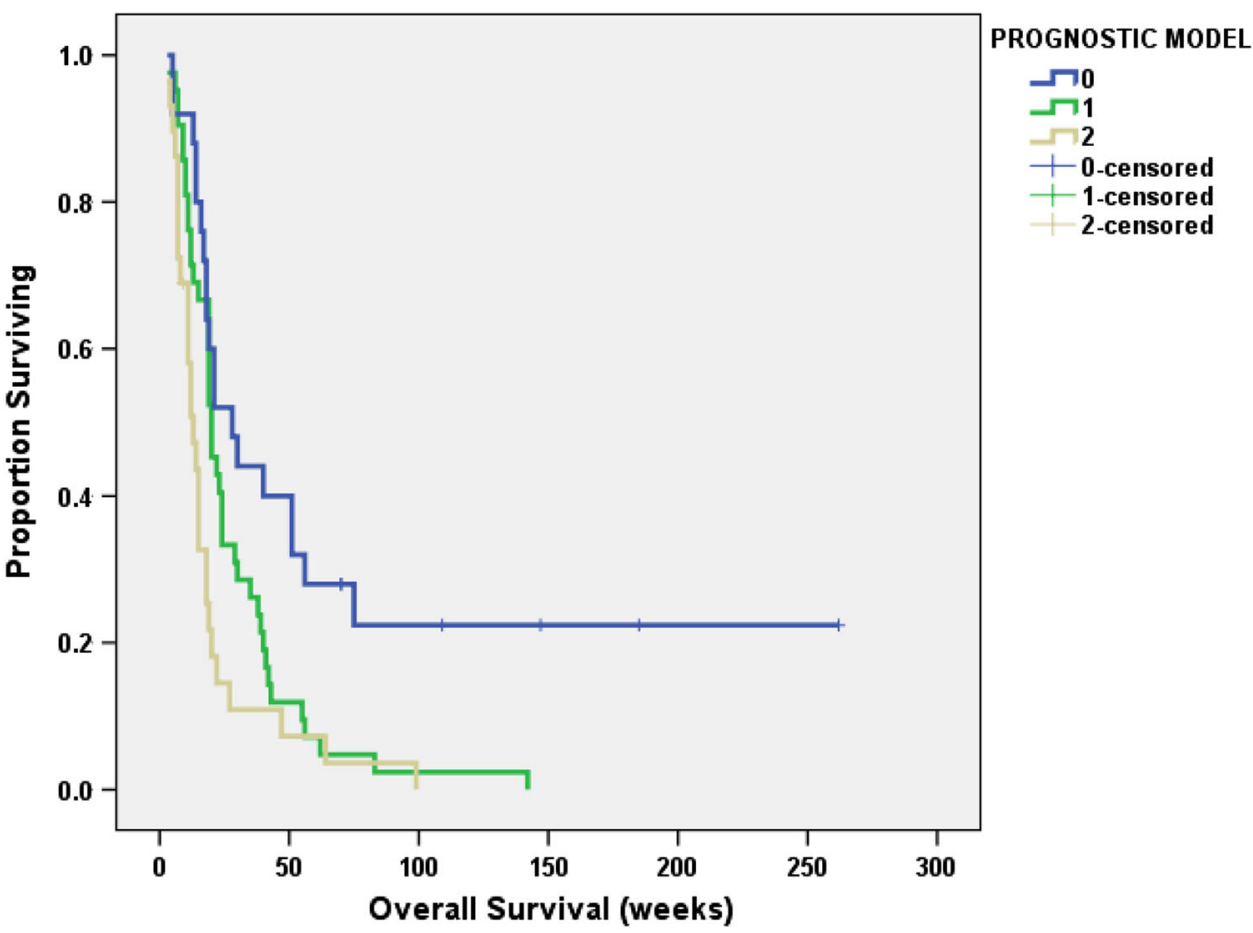


patients who achieved a PR with a single agent therapy also received an IGF-1R inhibitor.

Although we acknowledge the inherent limits of any inter-trail comparison, especially in the presence of a highly selected and heterogeneous population such as that recruited in our study, the outcome of patients receiving treatment in Phase I trials in the current series appears to be comparable to those observed in Phase III trials of chemotherapy versus best supportive care in the second line setting in UGIC. The overall median PFS and OS for the overall population were 7.7 and 19.1 weeks, respectively. Moreover, a significantly better outcome was observed in the group of patients who achieved a PR (median PFS and OS 28.0 and 51.0 weeks, respectively).

Our data demonstrates the largest examination of UGIC patients treated in a Phase I clinic to date. We describe a group of heavily pre-treated patients who had received a median of 2 prior lines of systemic therapy (range 1-4).

Considerable experience is needed in the selection of patients in order to meet the logistical and toxicological demands of Phase I trials; participation in such studies require frequent hospital visits and careful monitoring. To guide selection of UGIC patients for Phase I trial, we examined potential baseline variables that were prognostic of OS. In our series, only serum albumin and LDH were independently significant. In addition, these 2 variables had a moderate to good utility (area under the receiver operating characteristics curve of 0.710 (95 \% CI 0.601-0.819) in predicting mortality at 90-day (a commonly used inclusion criterion for Phase I clinical trials). As this is a single-centre retrospective study, further validation of our findings is clearly warranted. Nonetheless, these observations can augment clinical judgement when selecting potential upper gastrointestinal cancer patients for Phase I trials as well as provide data for discussion during medical consultations. Elucidation of the complex biology of such tumours coupled to increasing sophistication in the molecular profiling of tumours will hopefully facilitate the targeted treatment of UGIC. We anticipate that matching the targeted therapies with specific signal transduction abnormalities would be a rational way to improve on the modest efficacy observed so far in unselected patients as presented in this paper.

\section{Conclusion}

In conclusion, our analysis support the notion that Phase I clinical trials may represent a safe option for carefully selected patients with chemo-refractory UGIC, particularly when standard treatment options have been proven unsuccessful. It is however important for the clinicians to be aware that with experimental drugs in this setting, there is often risk of having potentially unforeseen toxicities and those should be carefully considered on a case-to-case basis when offering patient treatment within the context of Phase I studies.

Acknowledgments The Drug Development Unit of The Royal Marsden NHS Foundation Trust and The Institute of Cancer Research is supported in part by a program grant from Cancer Research UK. Support was also provided by the Experimental Cancer Medicine Centre (to The Institute of Cancer Research), the National Institute for Health Research Biomedical Research Centre (jointly to The Royal Marsden NHS Foundation Trust and The Institute of Cancer Research) and the Wellcome Trust (Grant 090952/Z/09/Z to Dr. Ang).

\section{References}

1. Siegel R, Naishadham D, Jemal A. Cancer statistics, 2013. CA Cancer J Clin. 2013;63(1):11-30. doi:10.3322/caac.21166.

2. Donaldson L, The UK. Public health system: change and constancy. Public Health. 2008;122(10):1032-4. doi:10.1016/j.puhe. 2008.05.001.

3. Philip PA, Mooney M, Jaffe D, Eckhardt G, Moore M, Meropol $\mathrm{N}$, et al. Consensus report of the national cancer institute clinical trials planning meeting on pancreas cancer treatment. J Clin Oncol: Off J Am Soc Clin Oncol. 2009;27(33):5660-9. doi:10. 1200/JCO.2009.21.9022.

4. Cunningham D, Allum WH, Stenning SP, Thompson JN, Van de Velde CJ, Nicolson M, et al. Perioperative chemotherapy versus surgery alone for resectable gastroesophageal cancer. N Engl J Med. 2006;355(1):11-20. doi:10.1056/NEJMoa055531.

5. Neoptolemos JP, Stocken DD, Bassi C, Ghaneh P, Cunningham D, Goldstein D, et al. Adjuvant chemotherapy with fluorouracil plus folinic acid vs gemcitabine following pancreatic cancer resection: a randomized controlled trial. JAMA J Am Med Assoc. 2010;304(10):1073-81. doi:10.1001/jama.2010.1275.

6. Wagner AD, Unverzagt S, Grothe W, Kleber G, Grothey A, Haerting J, et al. Chemotherapy for advanced gastric cancer. Cochrane Database Syst Rev. 2010;3:CD004064. doi:10.1002/ 14651858.CD004064.pub3.

7. Vincent A, Herman J, Schulick R, Hruban RH, Goggins M. Pancreatic cancer. Lancet. 2011;378(9791):607-20. doi:10.1016/ S0140-6736(10)62307-0.

8. Conroy T, Desseigne F, Ychou M, Bouche O, Guimbaud R, Becouarn $\mathrm{Y}$, et al. FOLFIRINOX versus gemcitabine for metastatic pancreatic cancer. N Engl J Med. 2011;364(19):1817-25. doi:10.1056/NEJMoa1011923.

9. Sultana A, Smith CT, Cunningham D, Starling N, Neoptolemos JP, Ghaneh P. Meta-analyses of chemotherapy for locally advanced and metastatic pancreatic cancer. J Clin Oncol: Off J Am Soc Clin Oncol. 2007;25(18):2607-15. doi:10.1200/JCO. 2006.09.2551.

10. Cunningham D, Starling N, Rao S, Iveson T, Nicolson M, Coxon $\mathrm{F}$, et al. Capecitabine and oxaliplatin for advanced esophagogastric cancer. N Engl J Med. 2008;358(1):36-46. doi:10.1056/ NEJMoa073149.

11. Bang YJ, Van Cutsem E, Feyereislova A, Chung HC, Shen L, Sawaki A, et al. Trastuzumab in combination with chemotherapy versus chemotherapy alone for treatment of HER2-positive advanced gastric or gastro-oesophageal junction cancer (ToGA): a phase 3, open-label, randomised controlled trial. Lancet. 2010;376(9742):687-97. doi:10.1016/S0140-6736(10)61121-X.

12. Moore MJ, Goldstein D, Hamm J, Figer A, Hecht JR, Gallinger S, et al. Erlotinib plus gemcitabine compared with gemcitabine alone in patients with advanced pancreatic cancer: a phase III trial 
of the National Cancer Institute of Canada Clinical Trials Group. J Clin Oncol: Off J Am Soc Clin Oncol. 2007;25(15):1960-6. doi:10.1200/JCO.2006.07.9525.

13. Chau I, Norman AR, Cunningham D, Waters JS, Oates J, Ross PJ. Multivariate prognostic factor analysis in locally advanced and metastatic esophago-gastric cancer-pooled analysis from three multicenter, randomized, controlled trials using individual patient data. J Clin Oncol: Off J Am Soc Clin Oncol. 2004;22(12):2395-403. doi:10.1200/JCO.2004.08.154.

14. Wesolowski R, Lee C, Kim R. Is there a role for second-line chemotherapy in advanced gastric cancer? Lancet Oncol. 2009;10(9):903-12. doi:10.1016/S1470-2045(09)70136-6.

15. Thallinger CM, Raderer M, Hejna M. Esophageal cancer: a critical evaluation of systemic second-line therapy. J Clin Oncol: Off J Am Soc Clin Oncol. 2011;29(35):4709-14. doi:10.1200/ JCO.2011.36.7599.

16. Ford H, Marshall A, Wadsley J, Coxon F, Mansoor W, Bridgewater JA, et al. Cougar-02: a randomized phase III study of docetaxel versus active symptom control in advanced esophagogastric adenocarcinoma. Presented in ASCO; San Francisco. J Clin Oncol. 2013;30(suppl 34):2012 (abstr LBA4).

17. Pelzer U, Schwaner I, Stieler J, Adler M, Seraphin J, Dorken B, et al. Best supportive care (BSC) versus oxaliplatin, folinic acid and 5-fluorouracil (OFF) plus BSC in patients for second-line advanced pancreatic cancer: a phase III-study from the German CONKO-study group. Eur J Cancer. 2011;47(11):1676-81. doi:10.1016/j.ejca.2011.04.011.
18. Kang JH, Lee SI, do Lim H, Park KW, Oh SY, Kwon HC, et al. Salvage chemotherapy for pretreated gastric cancer: a randomized phase III trial comparing chemotherapy plus best supportive care with best supportive care alone. J Clin Oncol: Off J Am Soc Clin Oncol. 2012;30(13):1513-8. doi:10.1200/JCO.2011.39. 4585.

19. Ulrich-Pur H, Raderer M, Verena Kornek G, Schull B, Schmid K, Haider $\mathrm{K}$, et al. Irinotecan plus raltitrexed vs raltitrexed alone in patients with gemcitabine-pretreated advanced pancreatic adenocarcinoma. Br J Cancer. 2003;88(8):1180-4. doi:10.1038/sj. bjc. 6600883 .

20. Boeck S, Weigang-Kohler K, Fuchs M, Kettner E, Quietzsch D, Trojan J, et al. Second-line chemotherapy with pemetrexed after gemcitabine failure in patients with advanced pancreatic cancer: a multicenter phase II trial. Ann Oncol: Off J Eur Soc Med Oncol/ ESMO. 2007;18(4):745-51. doi:10.1093/annonc/mdl463.

21. Custodio A, Puente J, Sastre J, Diaz-Rubio E. Second-line therapy for advanced pancreatic cancer: a review of the literature and future directions. Cancer Treat Rev. 2009;35(8):676-84. doi:10. 1016/j.ctrv.2009.08.012.

22. Ang JE, Olmos D, Moreno Garcia A, Brunetto A, PapadatosPastos D, Arkenau HT, et al. Utility of novel dynamic clinical indices in patients enrolled in Phase I oncology trial as markers of prognosis and treatment benefit. J Clin Oncol. 2011;29:Abstr 2555 . 